\title{
The immunopharmacology of antileprosy agents
}

\author{
R ANDERSON \\ Immunology Section, Department of Medical Microbiology, Insti- \\ tute of Pathology, University of Pretoria, PO Box 2034, Pretoria \\ 0001, Republic of South Africa
}

Received for publication 24 September 1982

\section{Introduction}

Unlike other acute and chronic bacterial infections, the antimicrobial chemotherapy of leprosy is complex due to the occurrence of adverse immunological reactions. The role played by antimicrobial agents in precipitating or exacerbating these reactions is controversial. Whilst conceding that various mechanisms of induction of adverse immunological reactions exist in this disease, the purpose of this review is to suggest immunopharmacological mechanisms by which chemotherapeutic agents may enhance immune reactivity in individuals with leprosy. To understand these mechanisms by which antimicrobial chemotherapy may contribute to immunologically mediated conditions it is necessary to consider the immunological status of untreated individuals with the lepromatous (LL) form of the disease.

\section{Immunological status of untreated individuals with $\mathbf{L L}$}

Acquired specific immunological unresponsiveness (tolerance, anergy) to Mycobacterium leprae antigens is found in individuals with LL and may be total or partial according to the state of advancement of the disease and bacillary load. This specific anergy develops as a consequence of the extremely high antigen load which occurs in LL. Apparently, the high antigen concentrations in vivo reach a threshold at which the host immune system detects that sustained immune reactivity against $M$. leprae is to the continued detriment of the host. In this situation the immune response is ineffective in eradicating the antigen, but continues to inflict damage on bystander tissues in the vicinity of the antigen. Immunologically mediated tissue damage occurs by the release of toxic oxygen radicals and proteolytic enzymes, such as elastase and collagenase, from phagocytic cells (Figure 1) which have been mobilized and activated by pro-inflammatory lymphokines released from antigen-activated T-lymphocytes.

Predisposition to the development of this chronic, ineffective inflammatory response which leads to LL may be genetically determined or acquired (e.g. in nutritional deficiency states). There is no conclusive evidence to indicate the existence of genetic susceptibility to the disease. Induction of 


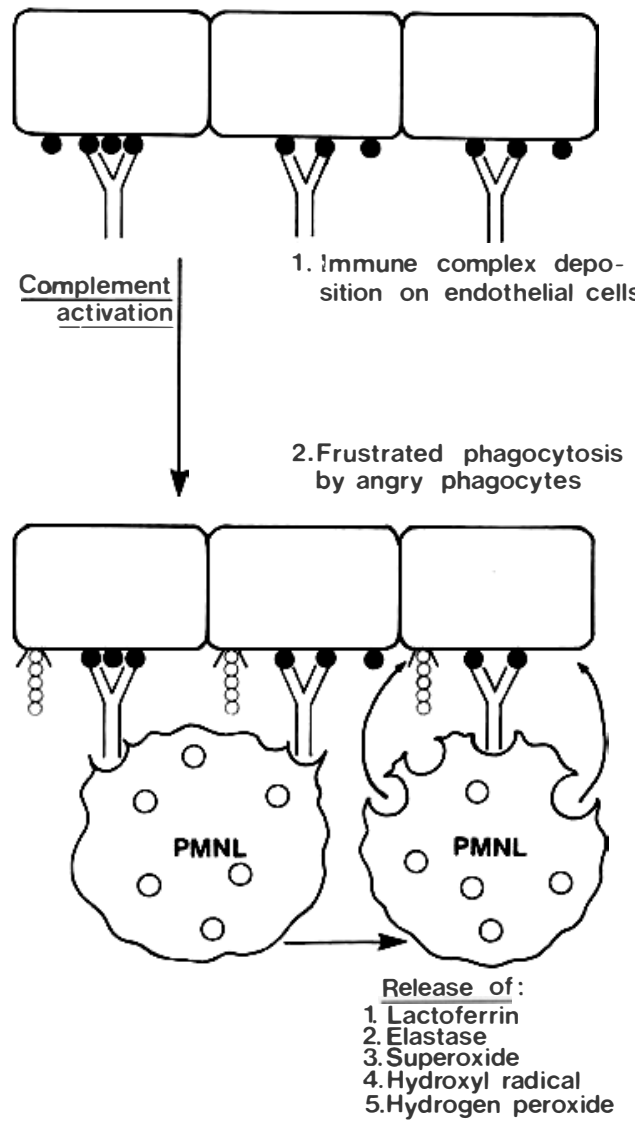

Figure 1. This is a diagrammatic representation of a possible mechanism of ENL assuming an immune complex pathology. $M$. leprae antigens $(\bullet)$ bind with antibody $(\gamma)$ to form localized or circulating immune complexes which are deposited in the skin, joints, kidney or endothelial cells of blood vessels. Resultant complement activation releases factors (C3a and $\mathrm{C} 5 \mathrm{a}$ ) which activate and attract phagocytes, especially PMNL and the cell membrane attack unit $\overline{\mathrm{C} 5 \mathrm{~b}, 6,7,8,9}$ (ooooo). These cells bind to the immune complexes but are unable to ingest them (since the complexes are tissue bound). The phagocytes therefore become 'frustrated' and 'angry' with consequent degranulation and release of toxic agents such as lactoferrin, elastase, the toxic oxidizing radicals superoxide and hydroxyl radical and hydrogen peroxide which probably cause the tissue damage in ENL. The mechanisms by which these agents mediate inflammation and tissue damage have been recently reviewed. ${ }^{19}$

tolerance is mediated by recruitment of antigen-specific suppressor T-lymphocytes ${ }^{1}$ which suppress specific cell-mediated immunity (CMI) to $M$. leprae. Other mechanisms which decrease CMI responses to $M$. leprae are also operative such as generalized anergy and humoral factors with immunosuppressive activity. Paradoxically, the induction of suppression of specific CMI is probably beneficial by reducing the degree of immunologically mediated tissue damage. However, damage to tissues is an on-going process due to non-specific and antibody mediated immune mechanisms and immunologically uncontrolled growth of $M$. leprae. 
The situation in individuals with L L prior to the commencement of antimicrobial chemotherapy is that they have: (a) an extremely high antigen load; and (b) specific immunological tolerance to $M$. leprae.

\section{Activation of immune reactivity in $\mathrm{LL}$ following antimicrobial chemotherapy}

Antimicrobial agents may contribute to the development of adverse immunological reactions by either or both of two possible mechanisms:

(a) As a consequence of the antibacterial activity of the drugs with activation of latent or hitherto suppressed immunological reactions. Antimicrobial agents cause disintegration of the bacterial cells with release of antigens which form circulating or localized immune complexes. These complexes cause regional or generalized complement activation with mobilization of granulocytes which migrate to sites of immune complex deposition. Binding of granulocytes to the immune complexes with subsequent phagocytosis or exocytosis causes release of toxic oxygen radicals and proteolytic enzymes which damage surrounding tissues. It is likely that granulocyte activation by the interaction of immune complexes and complement is responsible for the development of erythema nodosum leprosum (ENL) and its complications in individuals with a high bacillary load (BL-LL).

Antigen-elimination during antimicrobial chemotherapy causes a decrease in the antigen load with a consequent reduction in the extent of antigen-induced immunosuppression and recovery of specific CMI to $M$. leprae. Reactivation of CMI leads to the development of an adverse immunological reaction (reversal immunity reaction) caused by the induction of production of pro-inflammatory lymphokines which mobilize, attract and activate granulocytes, macrophages and T-lymphocytes. These highly reactive cells release toxic oxidants and proteases which, although important in the intracellular destruction of microorganisms, are also released extracellularly and may mediate the tissue damage which accompanies reversal immunity reactions. These reactions may occur anywhere in the leprosy spectrum except the polar groups.

It must be emphasized that these proposed mechanisms are speculative. However, should they exist all agents used in the antimicrobial chemotherapy of leprosy have the potential to cause ENL and/or reversal immunity reactions in susceptible individuals.

(b) The second mechanism by which antimicrobial agents may contribute to the development of adverse immunological reactions is by possession of intrinsic immunostimulatory activity, i.e. direct drug-mediated enhancement of cellular immune responsiveness independent of antimicrobial activity. Such a mechanism is probably less important than antigen release mechanisms related to antimicrobial activity. However, a drug such as dapsone which has been reported to increase granulocyte motility and lymphocyte proliferation ${ }^{2}$ could be expected to potentiate ENL and reversal immunity reactions in susceptible individuals.

\section{Effects of antileprosy drugs on cellular-immune reactivity}

The three widely used antimycobacterial agents rifampicin, dapsone, and clofazimine may regulate cellular immune functions by antigen-elimination mechanisms as described above. However, in this section their effects per se on immune reactivity are considered.

\section{RIFAMPICIN}

The immunomodulating effects of rifampicin have recently been reviewed. ${ }^{3}$ This antimicrobial agent is an inhibitor of lymphocyte responses to mitogens and antigens and of PMNL migration in 
vitro. Animal studies have also shown that rifampicin is immunosuppressive in vivo causing inhibition of both antibody and cell-mediated immune responses. However, studies ${ }^{4}$ have shown that rifampicin at concentrations of $0 \cdot 01-100 \mu \mathrm{g} / \mathrm{ml}$ had no effects on human monocyte migration in vitro. ${ }^{4}$ The effects of rifampicin on humoral and cellular immunity have been investigated ${ }^{5}$ in a double blind comparison in which 33 patients with pulmonary tuberculosis were treated with streptomycin, isoniazid and rifampicin or with streptomycin, isoniazid and pyrazinamide and 41 healthy controls were treated with rifampicin or a placebo. Treatment was for 6 months with a 1 year follow-up. No effects of rifampicin could be demonstrated on parameters of humoral or cellular immunity. In two separate studies we observed no inhibitory effects of rifampicin intake on polymorphonuclear leucocyte (PMNL) migration ${ }^{6,7}$ over a 1 -month period in individuals with LL and actually observed improved lymphocyte responsiveness to mitogens. The effects of rifampicin on humoral and cell-mediated immune responses appear to be variable according to the response studied and the in vivo model used. However, the presently available evidence suggests that ingestion of the antibiotic by individuals with LL and normal adults has no striking immunosuppressive effects. There is no evidence to show that rif ampicin per se stimulates any cellular immune function although improved lymphocyte proliferation in patients with LL may be associated with the antimicrobial activity of the antibiotic. ${ }^{7}$

\section{DAPSONE}

It has been reported from this laboratory that dapsone per se causes stimulation of PMNL motility in normal adults and individuals with LL in vitro. ${ }^{2}$ Furthermore ingestion of the drug over short periods was associated with increased PMNL migration and lymphocyte responsiveness to mitogens in the control and LL groups. ${ }^{2,6,7}$ These effects of dapsone were related to the anti-oxidant activity of the drug and not to its antimicrobial properties. Anti-oxidants sustain and enhance cellular immune reactivity by preventing the auto-oxidative loss of migratory responsiveness of PMNL and mitogen and antigen-induced lymphocyte proliferation. ${ }^{8} \mathrm{~A}$ second possible mechanism of dapsone-mediated immunostimulation, also related to an anti-oxidant mechanism, may be inhibition of the synthesis of immunosuppressive prostaglandins (PGs). Recent reports have indicated that PGs released by monocytes induce suppressor cell activity which may be the cause of the impaired CMI observed in diseases such as Hodgkin's disease. ${ }^{9}$ It has been reported ${ }^{10}$ that this PG-dependent suppression is operative in individuals with the BT and TT forms of the disease but not in the $\mathrm{BL}$ and $\mathrm{LL}$ forms. However, it is possible that during antimicrobial chemotherapy associated recovery of CMI in BL-LL cases that T-lymphocytes may become more responsive to PG-mediated suppression. Inhibition by dapsone of this mechanism may therefore possibly contribute to enhanced CMI and development of reversal immunity reactions. It must be stressed, however, that there is no available data to substantiate the existence of this mechanism.

These observations suggest that dapsone is pro-inflammatory and may contribute to ENL and reversal immunity reactions by stimulating P.MNL motility and lymphocyte responsiveness to antigens respectively. However, the drug has well-documented anti-inflammatory activity in a variety of dermatological conditions ${ }^{11}$ which is probably related to its ability to inhibit phagocyte degranulation. ${ }^{12}$ It has also been suggested ${ }^{13}$ that dapsone may confer a measure of protection against the development of reversal immunity reactions in individuals with BL. This may seem difficult to reconcile with the proposed pro-inflammatory activity of the drug in LL. However, in individuals with LL and a high antigen load it is possible that the immunostimulatory, pro-inflammatory activities of the drug are dominant since the anti-inflammatory effect on degranulation may be negated as a result of increased leucocyte infiltration and high concentrations of immune complexes. 
CLOFAZIMINE

Clofazimine, known alternatively as lamprene (R) or B663, is also a widely used antileprosy drug. However, clofazimine has no documented immunostimulatory properties and on the contrary has been reported to be usef ul in controlling both $\mathrm{ENL}^{14,15}$ and reversal immunity reactions ${ }^{15,}{ }^{16}$ whilst conferring antimicrobial chemotherapy. Recent investigations in this laboratory have shown that clofazimine inhibits the motility of PMNL and mitogen-induced transformation of lymphocytes from normal adults and individuals with LL in vitro; similar effects were observed following ingestion of the drug. ${ }^{17,18}$ These observations suggest that the most probable mechanisms of clofazimine-mediated anti-inflammatory activity are inhibition of PMNL migration and Tlymphocyte responsiveness to antigens which may control ENL and reversal immunity reactions respectively. Although the drug is therapeutically useful as a combined anti-inflammatory and antimicrobial agent its ability to precipitate adverse immunological reactions in susceptible individuals by antigen release mechanisms should, however, not be underestimated.

\section{Conclusions}

Inadvertent immunological manipulation occurs during antimicrobial therapy of individuals with leprosy with possible development of adverse immunological reactions in some cases. This is due to the formation of immune complexes and loss of antigen-induced immunosuppression and occurs as a consequence of the antimicrobial activity of the drugs. Rifampicin, dapsone and clof azimine may precipitate ENL and reveral immunity reactions by this mechanism. Dapsone-associated reactions may be intensified by the ability of the drug per se to potentiate PMNL migration and T-lymphocyte proliferation. Clofazimine, however, is immunosuppressive and may be usef ul in the control and prevention of such reactions whilst continuing to provide antimicrobial chemotherapy.

\section{References}

1 Godal T, Myrvang B, Froland SS, Melaku G. Evidence that the mechanism of immunological tolerance (central failure) is operative in the lack of host resistance in lepromatous leprosy. Scand J Imm, 1972; 1: 311.

2 Anderson R, Gatner EMS, Van Rensburg CE, Grabow G, Imkamp FMJH, Kok SK, Van Rensburg AJ. Invitro and in vivo effects of dapsone on neutrophil and lymphocytes functions in normal individuals and patients with lepromatous leprosy. Antimicrob Ag Chemo, 1981; 19: 495.

${ }^{3}$ Finch R. Immunomodulating effects of antimicrobial agents. J Antimicrob Chemo, 1980; 6: 691.

${ }^{4}$ Campbell PB. Defective leukotaxis in monocytes from patients with pulmonary tuberculosis. $J$ Inf Dis, 1979; 139: 409.

${ }^{5}$ Humber DP, Nsanzumuhire H, Aluoch JA, Webster ADB, Aber VR, Mitchison DA, Girling DJ, Nunn AJ. Controlled double-blind study of the effect of rifampin on humoral and cellular immune responses in patients with pulmonary tuberculosis and in tuberculosis contacts. Amer Rev Resp Dis, 1980; 122: 425.

${ }^{6}$ Anderson R, Gatner EMS. Changes in neutrophil motility accompanying dapsone and rif ampicin therapy. Lepr Rev, 1981; 52: 19.

7 Anderson R, Gatner EMS, Imkamp FMJH, Kok SH. In vivo effects of propanolol on some cellular and humoral immune functions in a group of patients with lepromatous leprosy. Lepr Rev, 1980; 51: 137.

8 Anderson R, Oosthuizen R, Grabow G. Prevention of peroxidase-mediated inhibition of neutrophil motility and lymphocyte transformation by levamisole, OMPI, sodium aurothiomalate, indomethacin and tolmetin in vitro. Int J Immunopharm, 1980; 3: 123. 


\section{$144 \quad R$ Anderson}

${ }^{9}$ Goodwin JS, Messner RP, Bankhurst AD, Peake JT, Saiki JH, Williams RC. Prostaglandin-producing suppressor cells in Hodgkin's disease. N Engl J Med, 1977; 297: 963.

10 Bahr GM, Rook GAW, Stanford JL. Prostaglandin-dependent regulation of the in vitro proliferative response to mycobacterial antigens of peripheral blood lymphocytes from normal donors and from patients with tuberculosis or leprosy. Clin exp Imm, 1981; 45: 646.

11 McDougall AC. Dapsone. Clin exp Dermat, 1979; 4: 139.

12 Lewis AJ, Gemmell DK, Stimson WH. The anti-inflammatory profile of dapsone in animal models of inflammation. Agents and Actions, 1978; 8: 578.

13 Barnetson RStC, Pearson JMH, Rees RJW. Evidence for the prevention of borderline leprosy reactions by dapsone. Lancet, 1976; ii: 1171 .

${ }^{14}$ Imkamp FMJH. A treatment of corticosteroid-dependent lepromatous patients in persistent erythema nodosum leprosum. A clinical evaluation of G30320 (B663). Le pr Rev, 1968; 39: 119.

15 Schulz J. 44 months' experience in the treatment of leprosy with clof azimine. Lepr Rev, 1971; 42: 178.

16 Pfaltzgraff RE. The control of neuritis in leprosy with clofazimine. Int J Lepr, 1972; 40: 392.

17 Van Rensburg CE, Gatner EMS, Imkamp FMJH, Anderson R. Effects of clofazimine alone or combined with dapsone on neutrophil and lymphocyte functions in normal individuals and patients with lepromatous leprosy. Antimicrob Ag Chemo, 1982; 21: 693.

18 Gatner EMS, Anderson R, Van Rensburg CE, Imkamp FMJH. The in vitro and in vivo effects of lamprene on the motility of neutrophils and transformation of lymphocytes from normal individuals. Lepr Rev, 1982; 53: 85.

19 Anderson R. Mediators of inflammation and tissue damage. S Afr Med J, 1982; 62: 365. 